This is the accepted manuscript of the article, which has been published in Ge S.S. et al. (eds) Social Robotics : The 10th International Conference, ICRS 2018, Qingdao, China, Nov 28-30, 2018. Dordrecht: Springer. Lecture Notes in Computer Science no. 11357. ISBN: 978-3-030-05203-4. ISSN: 0302-9743.

\title{
Robots and Human Touch in Care: Desirable and Non- Desirable Robot Assistance
}

\author{
Jaana Parviainen, corresponding (ORCID: 0000-0002-7838-592X), \\ Tuuli Turja (ORCID: 0000-0001-7815-9511) \\ Lina Van Aerschot (ORCID 0000-0001-8493-4166) \\ Faculty of Social Sciences, University of Tampere, Tampere, Finland \\ jaana.parviainen@uta.fi \\ tuuli.turja@uta.fi \\ lina.van.aerschot@uta.fi
}

\begin{abstract}
Care robots are often seen to introduce a risk to human, touch based care. In this study, we analyze care workers' opinions on robot assistance in elderly services and reflect them to the idea of embodied relationship between a caregiver, care receiver and technology. Our empirical data consists of a survey for professional care workers $(n=3800)$, including registered and practical nurses working in elderly care. The questionnaire consisted scenarios of robot assistance in care work and in elderly services and the respondents were asked to evaluate whether they see them as desirable. The care workers were significantly more approving of robot assistance in lifting heavy materials compared to moving patients. Generally, the care workers were reserved towards the idea of utilizing autonomous robots in tasks that typically involve human touch, such as assisting the elderly in the bathroom. Stressing the importance of presence and touch in human care, we apply the ideas of phenomenology of the body to understand the envisioned robot-human constellations in care work.
\end{abstract}

Keywords: Care robots; Elderly care; Human-Robot interaction; Touch; Triadic care

\section{Introduction}

Some of the critical voices have brought up concerns of care technologies and suggested that they may create of risk of dehumanizing and depersonalizing care and objectifying the care receivers by jeopardizing their individuality and subjectivity. For example, Barnard \& Sandelowski [1] have suggested that clinical and sterile environments characterized by standardization and strict regulation may fail to uphold and support human-centered care. In these kinds of environments with highly palpable and audible presence of equipment, people may sometimes become treated as extensions of the machinery. However, many care workers, nurses and caregivers welcome 
tools, techniques, equipment and robots that can assist them in work tasks, especially in physically demanding ones. Hence, there seems to be some tension between the ideals of 'touch-based' care and 'technology-driven' care, or 'humanistic' care and 'technologic frameworks' of care [2, 3].

We examine professional care workers' opinions on robot assistance in care work and specifically in elderly services. We introduce on one hand in which tasks robotic assistance is perceived as an acceptable idea and, on the other hand, care workers evaluations of undesirable robotic assistance in elderly care. In this paper, we will analyze these findings with a phenomenological approach and discuss the triadic relationship between a caregiver, care receiver and technology. Drawing upon the phenomenology of the body and Latour's [4] concept of ensemble, we develop a new approach to robot care as an embodied practice, triadic care.

\section{The Importance of Touching in Elderly Care}

Touching in care work is inevitable, because clients are dependent on nurses for many activities in daily living: washing, feeding, lifting, dressing, and other similar type of care activities that are related to the wellbeing and medical treatment of older, disabled or sick people. Care workers may use different forms of touch depending on their work tasks and communication with clients. Touching can be functional, purposeful and instrumental when lifting or feeding the client but still carry affective intentions, such as, comforting, reassuring and encouraging. Whether nursing touch is comforting, i.e. helping the patient to cope with the illness and its related stressors, or protective, protecting the patient from physical harm, the nurse's touch is supposed to be "professional touch". From the ethical point of view, professional touch refers to a special professional and ethical attitude in which the client's body is cared for and attended mindfully and respectfully but not too personal, emotional or intimate manners. Touch is also sharply separated from violence such as sexual abuse and harassment [5]. This implies that certain type of touching is considered appropriate in some social contexts and with some body parts, but decidedly inappropriate in others.

Body work is an essential part of care profession for it involves direct, hands-on activities, handling, assessing and manipulating bodies [6]. Profession- 
al touch in human care can take different forms. Depending on work tasks and social contexts, we can talk about instrumental touch [7], therapeutic touch [8] and expressive touch [9]. All tactile communication is reciprocal in nature: when a nurse touches a client s/he also being touched by the client [10]. Touching a lived body, a care worker reflects usually internally how her/his touch is being felt by the other body.

Being touched by others or being seen by others is considered especially crucial to the wellbeing of babies but also elderly people [11]. However, according to Langland and Panicussi [12], the more unable to communicate elderly people are due to, for example, memory disorders or other cognitive impairments, the more touch deprived they become. Yet, people with communicative or social restrictions often interpret feelings and affects that touching mediates and experience pleasure or displeasure within physical care practices [13].

Not all touching in care work is pleasurable for care workers or clients. In problematic situations-when a patient is violent, sexually aroused or psychotic - a care worker may need to call for colleagues or safeguards to help. In nurse-client relationships, feelings of disgust, shame, guilt or embarrassment are also common. These negative feelings are not seen to fit into the idea of professional nurse behavior. Some tasks like removing feces and changing diapers include bodily copresence [14]. These tasks can be felt disgusting but simultaneously raise feelings of empathy.

Touching becomes a more complex phenomenon when new technologies intervene in nurse-client relationships. Robotics for lifting patients out of their bed or into the bath, for example, do not necessarily mean that direct touching the patients has become more limited. New equipment may be used with a minimum of human effort but still require human presence to support, surveille or encourage the activity.

\section{Desirable and Non-Desirable Robot Assistance}

\section{Methods}

To analyze the acceptance of robot assistance in care tasks we used a survey data collected from professional care givers $(n=3800)$. The data was collected during the 
fall 2016 and was based on a random sample of Finnish elderly care workers. The questionnaire included multiple choice questions about educational and occupational background, experiences with assistive tools in healthcare and attitudes toward robots presented in a variety of care work scenarios. Regarding the work scenarios we used a question from used in Eurobarometer studies including also the response scale from 1 to 10 (see appendix A for specific questions). Assessing these scenarios respondents scaled firstly the usability of robotic assistance in care work $(\alpha 0.93)$ and secondly robotic assistance in elderly services. The latter were further categorized into autonomous robot assistance scenarios ( $\alpha$ 0.97) and tele-operated robot assistance scenarios ( $\alpha$ 0.95). We present our preliminary and descriptive results in percentages, means $(M)$ and differences between means $(t)$. The statistical difference between single assessments of robot-assisted work scenarios are observed by confidence intervals of 95 percent.

\section{Results}

Most of the respondents were women (95\%) working in public sector (78\%). They were typically practical nurses (56\%) or registered nurses (35\%), the rest being for example head nurses or physiotherapists (9\%). The age of the respondents varied from 17 to 70, the average being 46.5 years. Healthcare technology was fairly familiar to the respondents; safety phone to 71 percent, meal automaton to 11 percent, and Paro seal to 8 percent, to list few.

Care work consists a variety of tasks and physical labor is often a central part of the activities [15]. The questionnaire presented scenarios of care tasks performed or assisted by a robot. The variety of scenarios emphasized tasks that include body work. Firstly, respondents were to evaluate how comfortable they felt about the idea of robot assisting them in moving or lifting patients and heavy materials and also assisting them in threatening situations at work. Secondly, they were to evaluate how useful they perceive robot assistance in elderly care scenarios such as helping a physically impaired resident to move around in the home and in the bathroom.

The respondents were most comfortable with the idea of a robot helping them with physically straining work. Figure 1 shows that care workers were sig- 
nificantly more approving of robot assistance in lifting heavy materials compared to lifting patients. Regarding lifting or moving patients, the respondents were more comfortable with the idea of a separate robotic assistant compared to an exoskeleton for a worker to wear. However, moving patients using an autonomous stretcher was remarkably less welcomed compared to lifting patients with any robotic assistance. Summarizing these results, care workers see robots desirable primarily in other tasks than patient work. In addition, if robots are used in patient work, the care workers prefer situations where a care worker is present.

Care workers saw potential in robots assisting in threatening situations. This is not surprising as studies have shown that care workers have to endure and be prepared for aggression of patients and their close-ones [6, 16, 17]. In care scenarios touching is usually seen as something that is happening in care workers' terms. Here the respondents suggest that robotic applications could be also suitable in protective use where care workers are targets of unwanted contact.

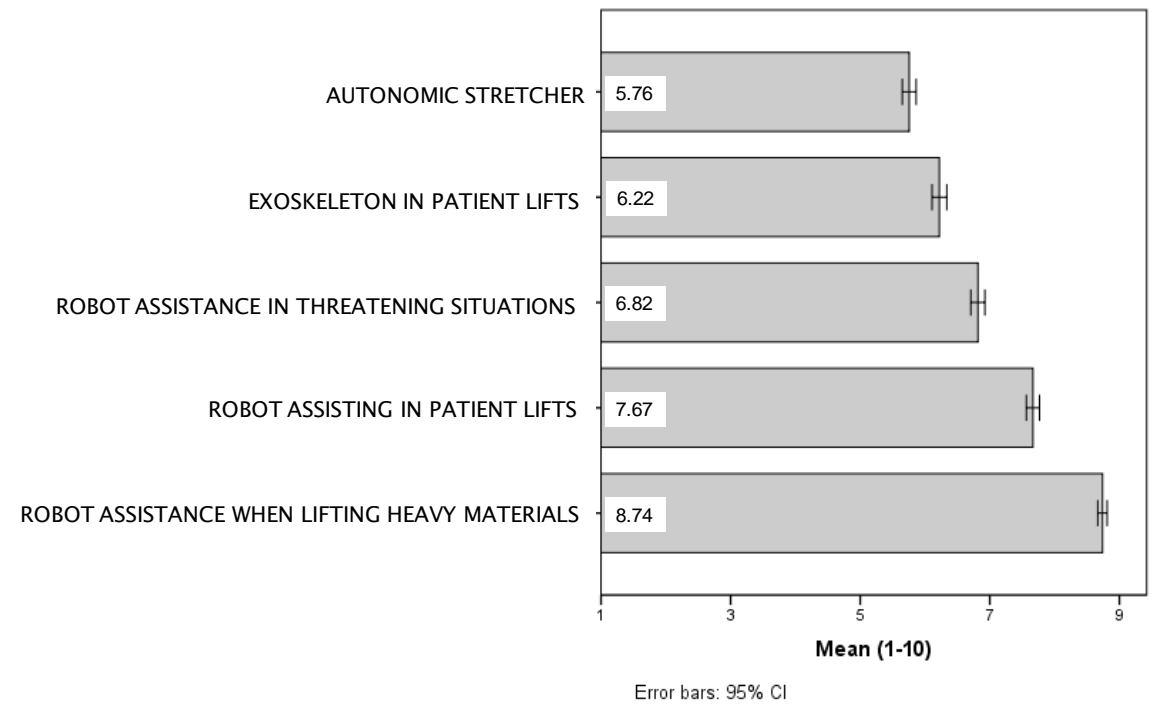

Figure 1. Acceptance of robot assistance at work, means on a scale from 1 to 10

When asked specifically about which elderly services could use robotic assistance, the respondents felt easier to see the benefits in tele-operated robots $(M=5.45)$ compared 
to autonomous robots $(M=5.16 ; t=-6.13 ; p<.001)$. Figure 2 presents the means for some of the scenarios. Out of these scenarios, care workers were mostly willing to see robots in situations where physical contact is not necessary, namely demonstrating light exercises to an elderly person. This kind of entertainment-like coaching by a robot was perceived more feasible than tele operated physiotherapy with a therapist. In addition, most of the respondents did not consider autonomous robots conducting physiotherapy suitable. The robotic assistance in bathing, dressing and in the toilet were met with a similar refusal. However, general support in moving around the residence was viewed more positively. Especially a robot which is remotely operated and monitored by care professionals could be used in the homes of older people as an assistants for moving, walking and getting up.

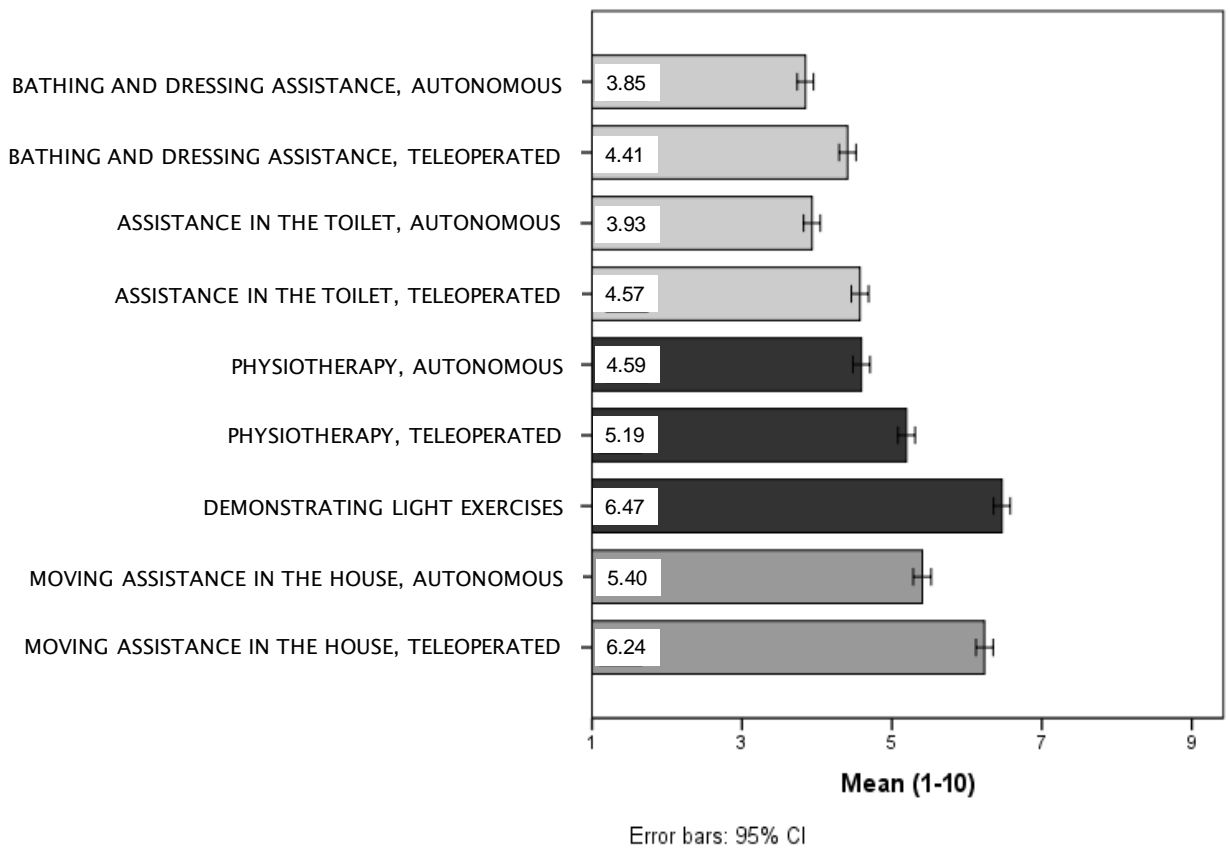

Figure 2. Acceptance of robot assistance in elderly care, means on a scale from 1 to 10

\section{Towards Triadic Care}

Care ethics is closely connected to professional touching and the physical presence of 
care workers with clients. Medical technologies have often been considered extensions of the nurse's body, but in the context of assisting robotics, the robot can be seen as a technological medium between the care worker and the client. Turkle [18] and van Wynsberghe [19] claim that embodied practices in human care, even if technologically assisted, always require a reciprocal interaction between the care-receiver and caregiver. Instead of focusing merely on the nurse-patient relationship, we call our approach "triadic care", which captures an idea of human-robot-human interaction instead of human-robot interaction. The notion of triadic care identifies the different roles of the care worker, care receiver and robot in care praxis. In the middle of this care triangle there are professional touching and embodied practices such as lifting, bathing, feeding, moving the care receiver and delivering medications/food/sheets to the room including social and cultural context of care settings. Different devices, tools and technologies can be used to assist in these tasks or even to conduct them autonomously but the devices are always in relation to the persons taking part in care giving and receiving activities.

The nurses' opinions on useful and acceptable robot assistance may be seen to oppose the ideas of standardized, technologized care and endorse the ideas of human dignity and individuality. Using here the triadic approach to care, nurses see robotics useful to distance and protect themselves physically from aggressive patients. They also consider robotic devices beneficial when assisting in physically demanding tasks of lifting patients and, especially, lifting heavy materials. In this way, more time and efforts might be saved for care tasks that include therapeutic touch, physical presence and support. An autonomic stretcher, however, draws more doubts. From the point of view of physical presence, a seemingly instrumental procedure of moving a patient from a ward to another in a stretcher can actually be a holistic event. Escorting a patient to an operation room, for example, is not just about the transport but a nurse may provide attention, comfort and encouragement by being present and able to touch.

Regarding robot assistance in elderly services, using a robot for personal care of assisting in bathing and dressing or going to the toilet is evaluated as the least acceptable scenarios. This kind of intimate assistance may be sometimes seen as being the core of care even though at the same time it may also entail negative mo- 
ments (i.e. assisting in toileting may provoke disgust).

The care workers opinions on robots assisting in their work and in tasks related to care of older people reflect the idea that technology should only be used in carerelated tasks which are not too intimate, affectionate and personal. The idea of triadic care is already present in the ways in which care workers think about robots: the care giver and care receiver make use of a technological devices in ways that suit to their needs without losing the possibility for human touch and interaction.

As stressed above, the touch involved in care practices transmits complex information about emotions and affects, creating a value-laden milieu [18]. In the context of triadic care, robotics is characterized as an interpersonal intervention that can develop a partnership and reciprocity in the nurse-client relationship.

\section{Discussion}

Identifying the significance of touch associated with the use of robots in elderly care is a necessary first step toward ethical discussions that can address senior persons' intimacy, individuality, autonomy, and rights to touch and being touched. More research is needed to examine how robotics will change nurses' working conditions and capabilities of using their touch in human care and to what extent can human touch be replaced by a robot.

Taking seriously the idea that touching and presence are crucial for the wellbeing of elderly people, we do not see the development of robots should aim at replacing caregivers. We suggest, as many other researchers [20, 21, 22], that robots should be designed to improve the quality of care rather than just to save money in the health care sector. When a care robots becomes a part of the network the distribution of roles and responsibilities as well as the care processes will change [19, 23]. If robotics does automate some of the tasks in human care, it is necessary to consider how to arrange mediating interdependencies within care relationships. van Wynsberghe [19] suggests an approach of value sensitive design and taking the ethical considerations as the first priority in the design process of care robots. She states that technologies are products of our culture and built on societal values and norms. Yet, technologies also change our culture and have far reaching impact on our socie- 
ties: "social norms, values and morals find their way into technologies both implicitly and explicitly and act to reinforce beliefs or to alter beliefs and practices” [19]. The use of independently functioning robots, even for some tasks, would fundamentally alter relations between caregivers and care receivers and nurses' care practices in elderly care.

\section{Literature}

1. Barnard, A. \& Sandelowski, M. (2001) The Shping of Organizational Routines and the Distal Patient in Assisted Reproductive Technologies. Nursing Inquiry 16(3), 241-250.

2. McConnell E.A. 1998. The coalescence of technology and humanism in nursing practice: It doesn't just happen and it doesn't come easily. Holistic Nursing Practice 12, 23-30.

3. May C. \& Fleming C. 1997. The professional imagination: narrative and the symbolic boundaries between medicine and nursing. Journal of Advanced Nursing 25: 1094-1100.

4. Latour, B. 1993. We Have Never Been Modern. Trans. C. Porter. Cambridge: Harvard University Press.

5. Paterson, M. 2007. The Senses of Touch: Haptics, Affects and Technologies. Oxford: Berg.

6. Twigg, J., Wolkowitz, C., Cohen, R.L. \& Nettleton, S. 2011. Conceptualising body work in health and social care. Sociology of Health \& Illness 33: 171-188.

7. Routsalo, P. 1999. Physical touch in nursing studies: a literature review. Journal of Advanced Nursing 30(4): 843-850.

8. Bullough, V.L. \& Bullough, B. 1998. Should nurses practice therapeutic touch? Should nursing schools teach therapeutic touch? J Prof Nurs. 14(4):254-7

9. Belgrave, M. 2009. The effect of expressive and instrumental touch on the behavior states of older adults with late-stage dementia of the Alzheimer's type and on music therapist's perceived rapport. Journal of Music Theraphy, 46(2):132-46.

10. Merleau-Ponty, M. 1968. The Visible and the Invisible, Followed by Working Notes trans. By A. Lingis. Evanston: Northwestern University Press.

11. Routasalo, P \& Isola, A. 1996. The Right to Touch and to Be Touched. Nursing Ethics 3: 73-84.

12. Langland, R. M. \& Panicucci, C. L. 1982. Effects of Touch on Communication with Elderly Confused Clients. Journal of Gerontological Nursing 8(3): 152-55.

13. Bush, E. 2001. The Use of Human Touch to Improve the Well-Being of Older Adults: A Holistic Nursing Intervention. Journal of Holistic Nursing 19(3): 256-70.

14. Wolkowitz, C. (2002) The social relations of body work, Work, Employment and Society, 16(3), 497-510. 
15. Cohen, R.L. 2011. Time, space and touch at work: body work and labour process (re)organisation. In J. Twigg, C. Wolkowitz, R.L. Cohen \& S. Nettleton (eds.) Body Work in Health and Social Care: Critical Themes, New Agendas. Blackwell.

16. Banerjee, A., Daly, T., Armstrong, P., Szebehely, M., Armstrong, H. and Lafrance, S. 2012. Structural violence in long-term, residential care for older people: Comparing Canada and Scandinavia. Social Science \& Medicine, 74(3):390-398. DOI: 10.1016/j.socscimed.2011.10.037.

17. Hintikka, N. \& Saarela, K. L. (2010) Accidents at work related to violence Analysis of Finnish national accident statistics database. Safety Science 48:4, 517-525.

18. Turkle, S. 2012. Alone Together: Why We Expect More from Technology and Less from Each Other. New York: Basic Books.

19. van Wynsberghe, A. 2013. Designing Robots for Care: Care Centered ValueSensitive Design. Science and Engineering Ethics 19: 407-33.

20. Jenkins, J. \& Draper, H (2015) Care, monitoring, and companionship: views on care robots from older people and their carers. International Journal of Social Robotics 7(5), 673-683.

21. Alaiad A, Zhou LN (2014) The determinants of home healthcare robots adoption: An empirical investigation. Int J Med Inf 83(11):825-840.

22. Kristoffersson A, Coradeschi S, Loutfi A, Severinson-Eklundh K (2011) An exploratory study of health professionals' attitudes about robotic telepresence technology. J Technol in Hum Serv 29(4):263-283.

23. Verbeek, P. (2008). Morality in design; design ethics and the morality of technological artifacts. In P. Vermaas, P. Kroes, A. Light, \& S. Moore (Eds.), Philosophy and design: From engineering to architecture (pp. 91-102). Berlin: Springer. 